\title{
A Relevant SNC Application for Data Computation using Python Programming
}

\author{
K Pooja, Shailaja. S
}

\begin{abstract}
Multiple applications of cloud servicing can be seen in the field of logical programming as well as IT industries. Complex computations over local machines may demand for plenty of system resources thereby delaying the data processing operations. In order to achieve speed in processing one must opt for cloud computing techniques. Extensive maneuver of cloud services is desirable for scientific computation of user data and application. This will require a platform designed in a way to meet the specific requirements of individual users, providing an ease for moving their data and applications over different devices. Symbolic-Numeric Computation using cloud service platform is presented in the paper. In this approach user tasks are presented in the form of symbolic expressions using languages like Java, C/C++, APIs etc. Proposed work employs Python programming for carrying out compilation process.
\end{abstract}

Key words: Python, Cloud computing, SNC, Tensor Flow, SVM.

\section{INTRODUCTION}

Countless services are offered by the cloud computing platforms ranging from storage of data and network resources securely to software and analytics. Web based interfaces helps the users to easily access these cloud services. Three types of cloud services are presented by the providers namely- Infrastructure as a Service (IaaS), Platform as a Service (PaaS) and Software as a Service (SaaS).

\section{A. Advantages of Cloud Computing}

$>$ Reduced cost: Overall cost of maintaining the complex servers and bulky datacenters is eliminated.

$>$ High Performance: Networking interfaces of invulnerable datacenters those are routinely upgraded to the recent versions are employed for the largest computations, thereby providing a few advantages against the solitary corporate data centers such as superior economies of scale and lowered latency of the networks.

> More reliable: The data can be stored on multiple networking sites offered by cloud services, thereby eliminating the threat of losing crucial data. As well as recovery and backing up process becomes inexpensive and effortless.

Manuscript received on September 19, 2021.

Revised Manuscript received on September 25, 2021.

Manuscript published on September 30, 2021

* Correspondence Author

K Pooja*, Department of Computer Science and Engineering, Poojya Dodappa Appa College of Engineering, Kalaburagi (Karnataka), India. Email: poojakbr09@gmail.com

Dr Shailaja S, Assistant Professor, Department of Computer Science and Engineering Poojya Dodappa Appa College, Kalaburagi (Karnataka) India. Email: shailajas@pdaengg.com

(c) The Authors. Published by Blue Eyes Intelligence Engineering and Sciences Publication (BEIESP). This is an open access article under the CC BY-NC-ND license (http://creativecommons.org/licenses/by-nc-nd/4.0/)
$>$ Security: Potential threats can be avoided by the use of large set of policies and controls included with cloud that will boost the overall safety.

Public, private and hybrid are the three basic types of cloud platforms. Privileges of parallel scientific applications comprises of dynamic provision and virtualization. In dynamic provision the resources required for processing the data will be issued vigorously unless they are no more required whereas virtualization enables the users to create their own specific clusters and workspaces in virtual environment hence offering on-demand data processing services for the demanding scientific computations. Anyhow barrier against the alluring scientific applications is the creation of feasible domain where efficient programming of complicated numerical algorithms and their deployment on different platforms of cloud can be carried out. This problem can be effectively addressed with the aid of SNC approach. Its features such as cross-platform interoperability and support for broad collection of languages make it predominant to other cloud platforms like SageMath and Wolfram platforms.

\section{LITERATURE SURVEY}

Paper [1] provides a vision of future times where computing may become an important utility for fulfillment of basic computing requirements like carrying out the crucial day to day requirements of common people. In order to achieve this perspective a lot of computing models are recommended with the newest one called as Cloud computing. In this work, researchers bring forth planning for forging Clouds by adapting resource allocations with the aid of manipulation of technologies such as virtual machines VMs. This paper [2] addresses the issue of performance ambiguity occurrence while processing of large business applications in an environment where the platform is accessed by multiple users simultaneously. This doubtfulness about higher performance variations of platforms cannot be cleared by the present SLAs, therefore an advanced utility design is proposed for attaining complete customer satisfaction. [3] Cloud computing services will expel the troublesome requirements of businesses and companies for fostering complex and expensive computing facilities. Taking into consideration the cloud features like dynamic resource sharing and virtualization, cloud offers a unique set of physical resources for accomplishment of various needs of a extensive user base.

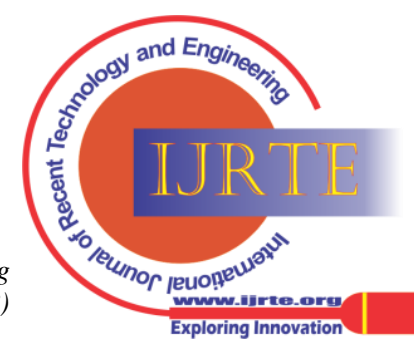




\section{A Relevant SNC Application for Data Computation using Python Programming}

Although the clouds provide its users with an advantage of economy of scale and facilities of grids, clusters to the scientists there are some limitations. The present day clouds are designed for handling the compact workloads of databases also the dynamic sharing and virtualization of complex database workloads will result in performance latency for scientific computing services. The researchers of this study examined this flaw and conducted an experimental evaluation of the performance of four cloud services inclusive of EC2 and Amazon the two supreme cloud platforms of present time.

[4] The extent of implementing private clouds for individual institutions is researched using a SciCloud project. Eucalyptus dependent private cloud and multiple personalized images are developed which will aid in eliminating the problems ranging from bio- informatics domains to distributed computing.

[5] Cloud platforms supports the on demand services of the customers by offering them a solitary long term subscription facility incapable of delivering the quality services thereby resulting in wastage of resources. A dual resource subscription strategy is discussed in this paper offering both shirt term and long term renting facilities thereby reducing the resource exhaustion and providing quality services.

[6] In spite of the concrete security and challenging models included by cloud platforms they often come under the threat of cyber attacks. This is the one main reason for which users hesitate to use cloud platforms for saving their precious application data. Thus a structured firewall is necessary to safeguard the cloud from such attacks and improve the usage of cloud services. A distributed firewall structure is offered by service providers and positioned in the middle of Internet interface and data centers of cloud. Firewall will also provides the facility of detecting any malware present in the file at the time of uploading it to cloud and alerts the users about it.

[7] Presents the journal proceedings about the administration of EC2 for scientific computing applications. In scientific computing approaches compact and resolute clusters will undoubtedly provide for low cost and sustainability, however not much strong for real time applications. The idea of virtual cluster is expanded by the largest cloud platform that is Amazon's Elastic Computing Cloud (EC2). Such a facility will offer the users with storage support and CPU power upon an on-demand request, facilitating the user to develop their own dedicated and dynamic computer clusters whenever required.

\section{EXISTING SYSTEM}

A few implementations claim to offer the processing of symbolic expressions using JIT compilation but they are unreal and will make use of two different approaches in order to carry out statistical estimations of symbolic expressions. In the first approach the symbolic expressions are identified and converted into transitional representation. This is then fed to the interpreter tool to execute the assessments. Some of the interpreters in use are Matlab's Function, a virtual machine by Mathematica and SageMath's interpreter. In the other approach, a source code is initially generated by making use of $\mathrm{C} / \mathrm{C}++$ /FORTRAN, next compiled using an AOT compiler and finally the compiled binary code is linked to the software environment. Few such examples are SymPy'sufuncify function and Theano's function. Speedy compilations are achieved in SNC with the aid of JIT compiler. Extremely improved machine codes operate instantly after the compilation. The duration required for evaluation of symbolic expressions is relatively reduced compared to the other implementations.

A cloud platform for SNC is idealized in this paper. Wide range of programming languages like JIT, python and Java are supported in SNC approach. Research is carried out using python programming.

\section{A. Hardware and Software Requirements of the Project}

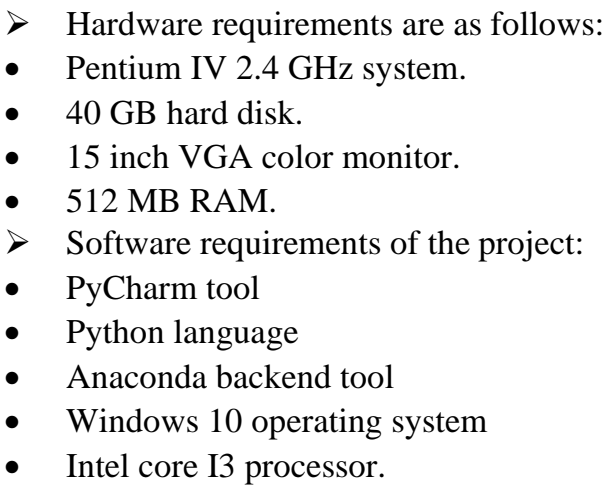

\section{DESIGN METHODOLOGY AND SYSTEM IMPLEMENTATION}

\section{A. System Design}

Fig.2 shows the data flow diagram of SNC. Initially the datasets are uploaded to the cloud server by the users. Soon as the data is uploaded to cloud platform it is divided into training and testing data. Testing data is used for carrying out numerical computations over cloud. The various algorithms of machine level learning are performed one by one. In order to check the suitability of algorithm to carry out symbolic numeric computations, the data is processed by every individual algorithm. The precision and accuracy of every algorithm is then compared. Cloud server makes a socket connection as soon as it gets started and will keep on checking constantly for the user to initiate the symbolic numeric computations. The numerical computations performed are represented at the background of the terminal. To establish the connection between cloud and the client, the user credentials are mandatory to be uploaded on the server. In the presented work the health conditions of a patient such as age, sugar, bp are provided in the form of data set. Binary values 0 and 1 are assigned to each condition. Occurrence of 0 indicates no health issues in patients whereas occurrence of 1 indicates the presence of some sufferings. The server site offered by cloud platform performs all this numerical computations providing the users with end results. An input port present on the cloud server will receive all the incoming datasets to be processed.

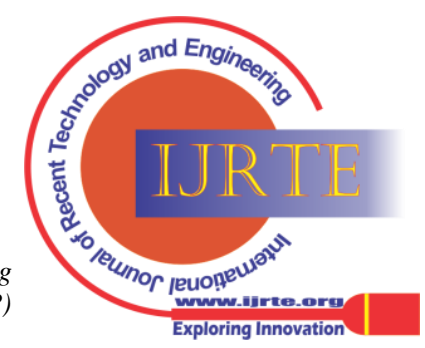




\section{Uploading Dataset}

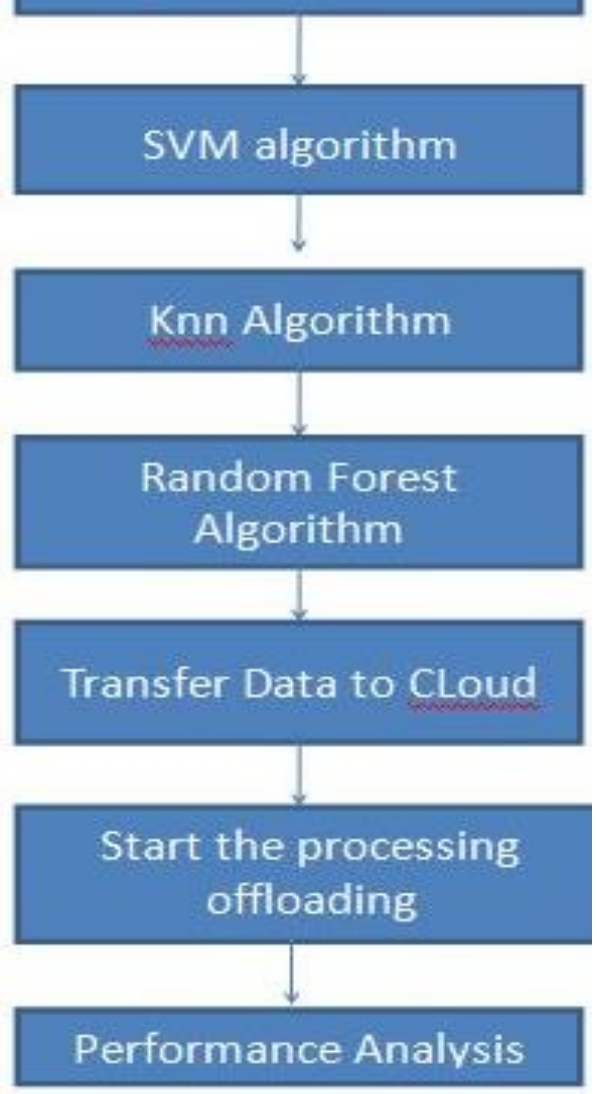

Fig. 1: Data Flow Diagram for SNC.

\section{B. Illustration of modules}

$>$ Client- Server computing: Programs executing on the servers are controlled by the service providers.

$>$ Cloud computing: Programs and software database of various users is continuously processed over the virtual machines by the cloud vendors. Computing cycles are offered by the vendors which will enable fast computations on user devices thereby saving energy. This energy saving mechanism is known as computation offloading.

> Virtualization: This process provides the facility of processing database of distinct users on distinct machines, offering protection and separation of individual data.

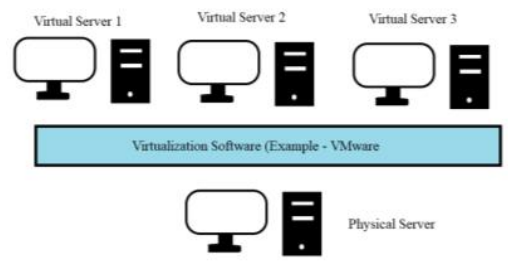

Fig. 2: Virtualization process

$>$ Intermediate processing: Series of algorithms compiled internally will convert links of the provided network into a series of compilation units. Individual layers are connected to separate compilation units. Nevertheless at some instances taking into consideration the advantages associated with the performance of generated code and compilation process, the layers might be partitioned into more than one compilation unit or they can be merged together as required. Further memory allocation is done for various input and output nodes, keeping in consideration the fact that unique memory must be assigned to tensors exhibiting overlapping lifetimes. During this phase, any memory requirements for outputs of each compiler layer will be indicated. The memory of input tensors which remain unreferenced will be allocated to compiler outputs. In a way like this, myriad compilers can be in operation simultaneously, resulting in finer utilization of cache as well as reduced operations of pointer register.

$>$ Activation functions: In semantic networks, the different mechanisms working on individual input elements independently are known as activation functions. Often yet independent to the location of elements inside the input tensor. Hence they can be simultaneously implemented keeping the addresses of their outputs and inputs in memory location unaltered and at the same time they can be used in other functions such as convolutional and completely linked layers. In such scenario, in order to prevent an extra loop of load and store operations, the activation functions are first made operational ahead of adding to the memory the payoff of the operations.

> Tensorflow: In order to obtain rapid accomplishment of programs with greater flexibility and programmability, tensorflow together with Just in Time (JIT) compilation is used. Compilation takes place during the time of execution in order to progressively compile the presumed tensorflow code into a productive and advanced machine code. Vigorous compilation will provide the tensorflow runtime with adequate data regarding the target code so that the programmer is not required to provide the details of its nature then and there.

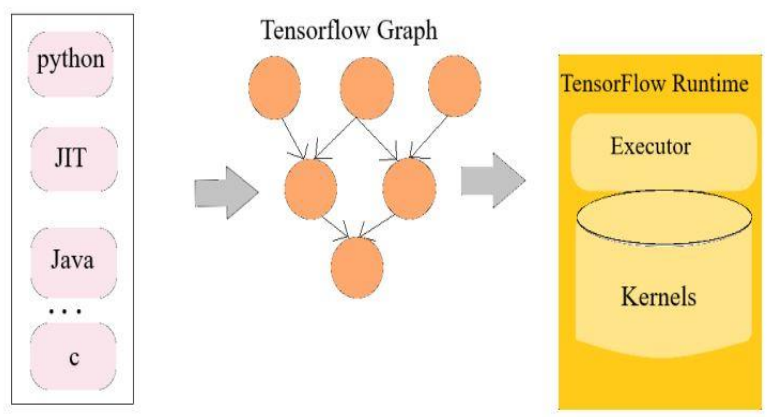

Fig. 3: Tensorflow operations.

\section{System Implementation using Python}

Python's syntax, along with dynamic computation and deciphering quality, make it an ideal language for planning and rapid application development. Python supports a variety of programming paradigms including objectoriented, fundamental, and practical or procedural programming. Python is not just intended to be used in a certain area.

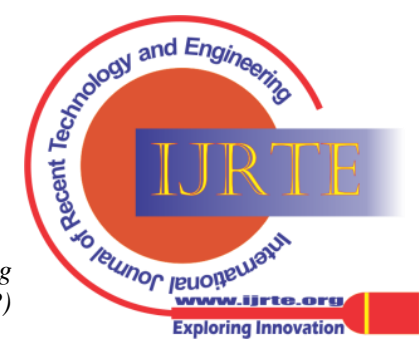




\section{A Relevant SNC Application for Data Computation using Python Programming}

That is why it is known as a multi-purpose programming language, as it can be used for web, enterprise, 3D, CAD and so on. Since there is no accumulation phase retained for python improvement and the test-troubleshoot cycle changes rapidly, python makes the turn of events and investigations swift. Undoubtedly Python is a broadly useful programming language. Its preplanning underscores the code readability and a linguistic structure that permits developers to communicate ideas in fewer lines of code than any other programming such as $\mathrm{C} / \mathrm{C}++$ or Java. It empowers error free programming on both small and large scopes. Python highlights a dynamic type system and automatic memory on board. It upholds multiple programming standards including procedural, object-oriented, functional and imperative and has a huge standard library.

\section{EXPERIMENTAL RESULTS AND DISCUSSION}

The dataset to be processed is uploaded on the graphical user interface of cloud enabling the cloud platform to perform numerical computations and send the result back to client. A number of algorithms are checked to select the better one for symbolic numeric computations. Graphical user interface of cloud server can be seen in fig. 5 .

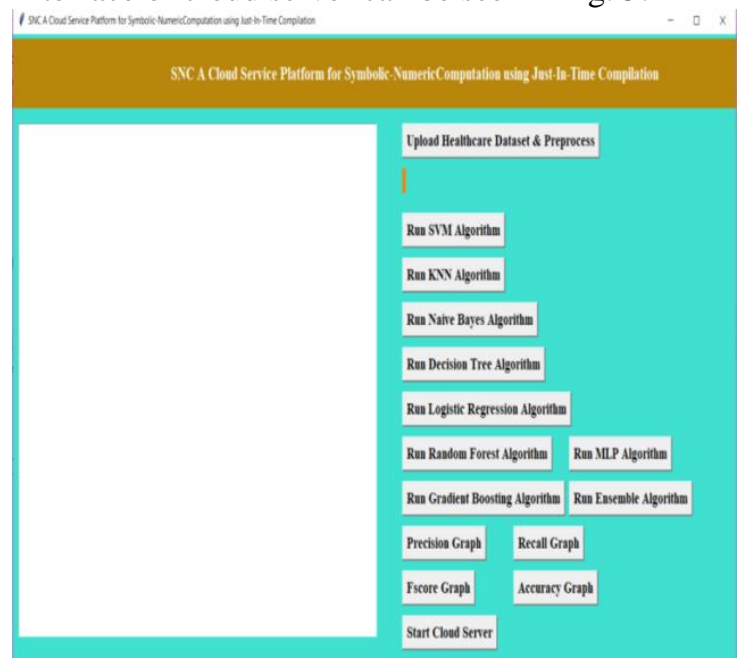

Fig. 4: GUI for Cloud Server

SVM features like Precision, Recall, Fmeasure and Accuracy are noted by running SVM algorithm as shown in fig. 6 .

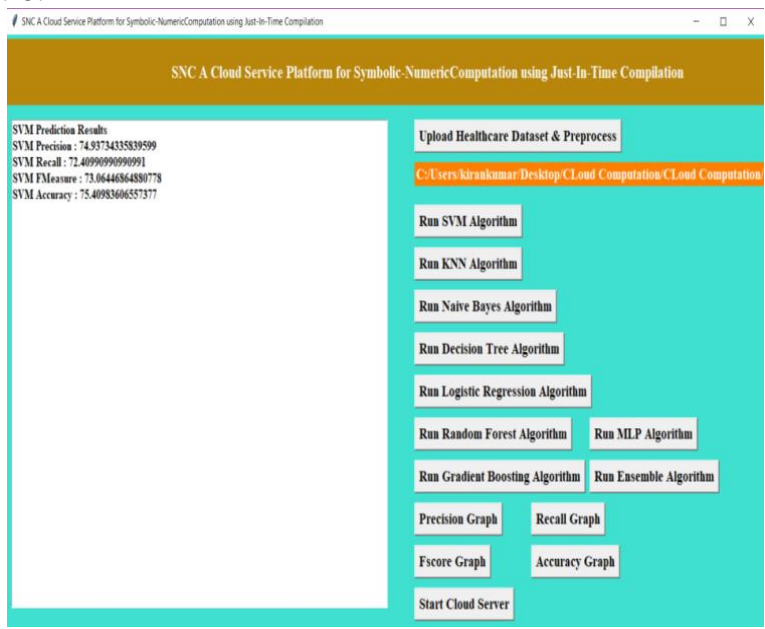

Fig. 5: SVM running procedure
Fig. 7 shows the graphical user interface for client device. GUI for client indicates where the data is required to be uploaded in order to get the appropriate results. As soon the data uploading is done the compilation process will be run.

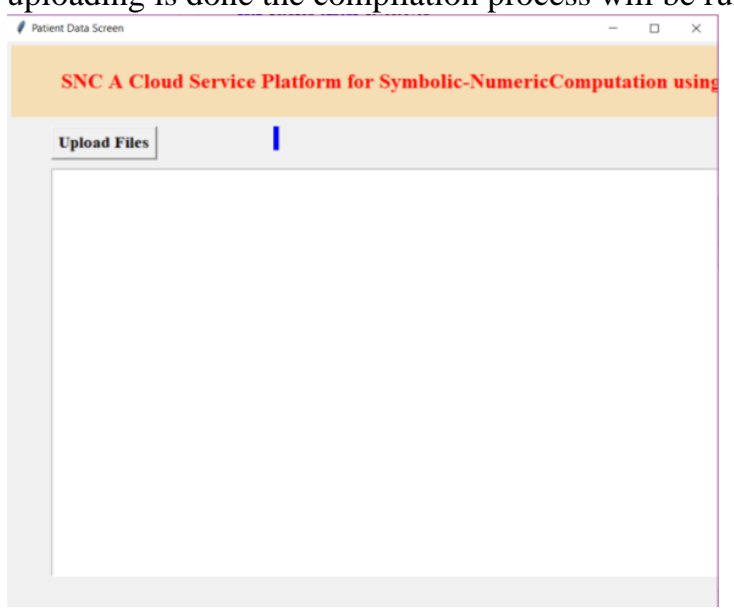

Fig. 6: GUI for Client

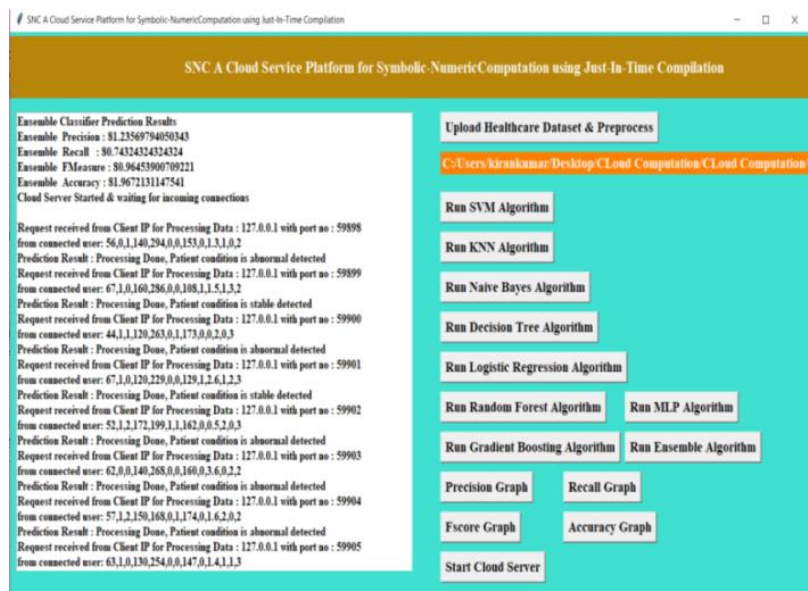

Fig. 7: Display of Results

All the details of the patient are shown whether the patient is normal or abnormal. Here the each patient is given a particular port number to upload their data for compilation. Fig. 9 displays where the compilation occurs of the given data of the clients and predicts the results. At the end it will have either 0 or 1 . 0 means it is not having any problem in the patients and 1 means patient is suffering with some health issues.

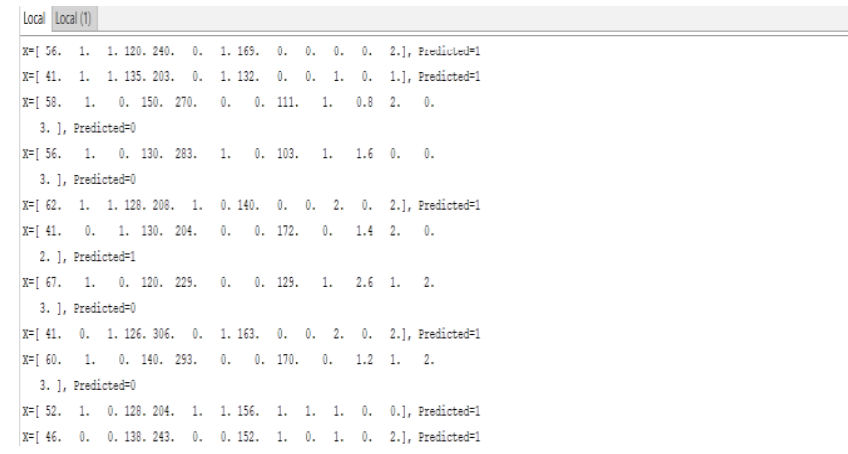

Fig. 8: Compilation Results

Published By:

Blue Eyes Intelligence Engineering and Sciences Publication (BEIESP)

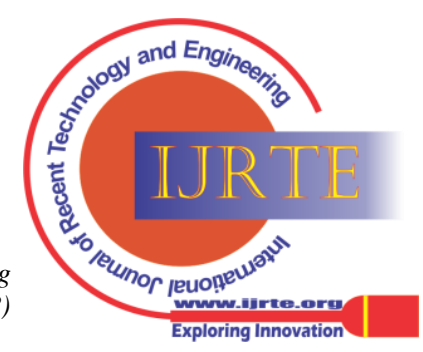




\section{CONCLUSION}

The proposed work introduced a Cloud administration stage for representative numeric calculations, the SNC stage and taken the case of the medical care of the patient, by taking the subtleties of the client the symbolic numeric computations are done on the cloud and afterward the cloud outputs the results to the user about their wellbeing or illness. Python aggregation happens at execution time to powerfully arrange deciphered TensorFlow code into profoundly proficient, upgraded machine code.

The results of work showed that:

$>$ The SNC stage speeds up the symbolic numeric computations.

$>$ This cloud based help stage empowers lightweight gadgets or cell phones to perform complex mathematical calculations.

\section{REFERENCES}

1. R. Buyya, C. S. Yeo, S. Venugopal, J. Broberg, and I. Brandic, "Cloud computing and emerging IT platforms: Vision, hype, and reality for delivering computing as the 5th utility," Future Generation Computer Systems, vol. 25, pp. 599-616, Jun 2009.

2. J. Chen, C. Wang, B. B. Zhou, L. Sun, Y. C. Lee, and A. Y. Zomaya "Tradeoffs between profit and customer satisfaction for service provisioning in the cloud"

3. Alexandrulosup, Member, IEEE, Simon Ostermann, M. NezihYigitbasi, Member, IEEE, RaduProdan, Member, IEEE, Thomas Fahringer, Member, IEEE, and Dick H.J. Epema, Member, IEEE, "Performance analysis of cloud computing services for manytasks scientific computing," Parallel and Distributed Systems, IEEE Transactions on, vol. 22, pp. 931-945, 2011.

4. SatishSrirama, Oleg Batrashev and EeroVainikko Distributed Systems Group Institute of Computer Science, University of Tartu J. Liivi 2, Tartu, Estonia \{srirama, olegus, eero\}@ut.ee "SciCloud: Scientific Computing on the Cloud"

5. Jing Mei, Kenli Li, Member, IEEE, AijiaOuyang and Keqin Li, Fellow, IEEE "Quality of Service in Cloud Computing"

6. Deepika A, BPrabadevi B, A Student, School of Information Technology and Engineering, VIT University, India B Assistant Professor School of Information Technology and Engineering, VIT University, India Adeepikaanandms@yahoo.com, Bprabadevi.b@vit.ac.in

7. S. Hazelhurst, "Scientific computing using virtual high-performance computing: a case study using the Amazon elastic computing cloud," in Proceedings of the 2008 annual research conference of the South African Institute of Computer Scientists and Information Technologists on IT research in developing countries: riding the wave of technology, 2008, pp. 94-103.

\section{AUTHORS' PROFILE}

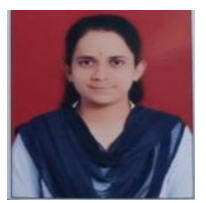

K Pooja, was born in Karnataka, India. She received her engineering degree from Poojya Dodappa Appa College of engineering Kalaburagi and pursuing her Masters in Computer Science from the same college.

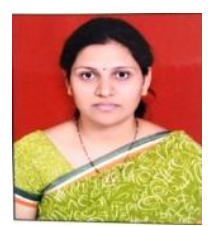

Dr. Shailaja S, obtained her B.E degree in the field of Computer Science and Engineering from VTU in 2005 and degree in M. Tech in the field of CSE from VTU in the year 2009. She completed her PhD from VTU as well. She is working as assistant professor in CSE Department, PDACE. She has published many papers in international journals and indeed a member of the Indian Society of Technical Education.

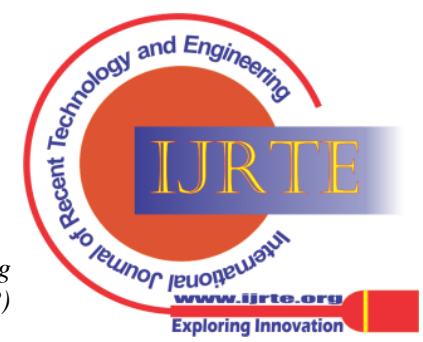

